\title{
ANALYTICAL PROCEDURE ELABORATION OF TOTAL FLAVONOID CONTENT DETERMINATION AND ANTIMICROBIAL ACTIVITY OF BEE BREAD EXTRACTS
}

\author{
NATALIIA HUDZ ${ }^{*}$, OKSANA YEZERSKA', OLGA GRYGORIEVA², SOŇA FELSÖCIOVÁ ${ }^{3}$, \\ JÁN BRINDZA ${ }^{4}$, PIOTR P. WIECZOREK ${ }^{5}$ and MIROSLAVA KAČÁNIOVÁ ${ }^{3,6}$
}

${ }^{1}$ Department of Drug Technology and Biopharmaceutics, Danylo Halytsky Lviv National Medical

University, Pekarska street 69, 79010, Lviv, Ukraine

${ }^{2}$ M.M. Gryshko National Botanical Garden of Ukraine, National Academy of Sciences, Timiriazevska 1, 01014 Kyiv, Ukraine

${ }^{3}$ Faculty of Biotechnology and Food Sciences, Slovak University of Agriculture in Nitra, Slovak Republic

${ }^{4}$ Institute of Biodiversity Conservation and Biosafety,

Slovak University of Agriculture in Nitra, Slovak Republic

${ }^{5}$ Faculty of Chemistry, University of Opole, Opole, Poland

${ }^{6}$ Faculty of Biology and Agriculture, University of Rzeszow, Rzeszow, Poland

\begin{abstract}
Sixteen ethanolic extracts were obtained from seven different bee bread samples. The total flavonoid content in the extracts was determined by aluminium-chloride method and was in the range of $8.3 \mathrm{mg} / \mathrm{L} \pm$ $6.24 \%$ to $195.3 \mathrm{mg} / \mathrm{L} \pm 1.35 \%$ and $28.8 \mathrm{mg} / \mathrm{L} \pm 19.33 \%$ to $603.3 \mathrm{mg} / \mathrm{L} \pm 4.64 \%$ with reference to quercetin and rutin, respectively. The relative standard deviations (RSD) for parallel measurements for the calibration curves of quercetin dehydrate and rutin trihydrate were in the range of $0.51 \%$ to $9.39 \%$ and $5.02 \%$ to $19.91 \%$, respectively. The RSD for parallel measurements for the extracts with reference to quercetin dihydrate and rutin trihydrate were in the range of $0.23 \%$ to $11.64 \%$ and $4.64 \%$ to $19.33 \%$, respectively. The total flavonoid content mainly depended on a ratio of bee bread to $50 \%$ ethanol and technology of obtaining bee bread (presence or absence of drying operation). The increase of bee bread in the solvent in 2 times yield an elevation of the total flavonoid content in approximately 1.5 times at similar maceration time ( 7 days). The significant differences between results were statistically confirmed. Antimicrobial activity was evaluated with disc diffusion method. The best antibacterial activity of the bee bread extracts was found against Bacillus cereus CCM 2010, Clostridium perfringens CCM 4435, and Staphylococcus aureus subsp. aureus CCM 4223. The activity of the bee bread extracts against Gram-negative bacteria, Aspergillus and Penicillium genera was lower with moderate anticandidal activity. The obtained results indicated that it was very important to employ extracts with a high content of bee bread in 50\% ethanol $(1: 5,1: 10)$ during the development of bee bread extracts with the purpose of their potential usage in medical practice. According to the results of this study, bee bread is a product which is rich in flavonoids and with good antibacterial activity against Gram-positive bacteria and can be considered as a raw material for the development of diet supplements and antimicrobial medicinal products.
\end{abstract}

Keywords: bee bread, extracts, flavonoids, antimicrobial activity

\section{INTRODUCTION}

Bee bread is a fermented mixture of honey, pollen collected by bees and digestive enzymes of bee saliva. Bee bread is the main food of bee larvae and the main source of proteins in a bee's diet. (1-6). Bee bread has been used for many years in traditional medicine, supplementary nutrition and alternative diets, primary due to its nutritional properties and health benefits ( 6 , 7). Bee bread can be considered a food supplement due to a high content of nutrients (8). One of the contributions to its high nutritional value is the presence of significant amounts of phenolic compounds (flavonoids $(0.25-1.4 \%)$ and phenolic acids) as natural antioxidants, carbohydrates $(24.40-34.80 \%)$, proteins (20.2$26.0 \%)$, lipids $(0.67-4.5 \%)$, unsaturated and saturated

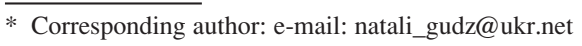


fatty acids, vitamins, free amino acids, $\mathrm{C}_{21}-\mathrm{C}_{35}$ alkanes, unsaturated alcohols, carotenoids, lactic acid (3.06-3.20\%), aliphatic acids, phytohormones, growing stimulators, etc. $(3-7,8,9)$. The potential application of bee bread as a food and nutriceutical supplement mainly depends on its chemical composition which varies directly with the flora of a region, geographic origin, climatic conditions, soil type, the time and year of pollen collection by bees, bee species, method of obtaining bee bread (presence or absence of drying operation) and storage conditions $(5,6,8)$. It was set up that polyphenols have a strong impact on the antioxidant activity of bee bread extracts (1-3, 5-9).

Bee bread has antimicrobial, antioxidant, hepatoprotective, immunomodulating, anti-inflammatory and antiradiation activity, adaptogenic properties, etc. $(1,2,7,10)$. It stimulates the protective forces of the human body, normalizes metabolism, has a positive influence on the liver, nervous and endocrine system functions, and enhances regeneration of tissues, physical and mental persistence of the human body $(2,7,9-15)$.

Phenolic acids and flavonoids as secondary metabolites are widespread in the plant kingdom $(10,16)$. Polyphenols are also components of bee pollen $(2,3,7)$. Flavonoids and phenolic acids possess antioxidant features, neutralize active oxygen species and scavenge free radicals due to their specific chemical structure and are associated with a reduced risk of various chronic diseases related to oxidation stress $(3,7)$. Among such diseases are cardiovascular diseases, cancer, diabetes, atherosclerosis, cataracts, cognitive dysfunction, and age-related functional decline, etc. $(12,16)$.

Flavonoids are present in pollen mainly in the form of glycosides, from which flavonol glycosides are present in the largest amount (7). The presence of a glycoside bond reduces antioxidative properties because of steric effects (7). The main flavonols of bee pollen are quercetin and kaempferol and their glycosides $(5-7,12,14)$. The level of free aglycones is a better indicator of pollen loads quality than the free amino acids content (7). The flavonoid rutin (quercetin - 3-O rutinoside) is a flavonol glycoside comprising the flavonol quercetin and disaccharide rutinose. Among other flavonoids are chrysin, apigenin, luteolin, etc. $(3,5-7,10,12,14,15)$. According to Chang C.C. et al. (17), Meda A. et al. (18) and Zuluaga C.M. et al. (3), the content of flavonoids is considered to be an important index for evaluating propolis, honey and bee bread quality, respectively.

There are few studies regarding the development of bee bread extracts with RSD analysis, repeatability (intra-assay precision) and intermedi- ate precision of an analytical procedure of the determination of total flavonoid content in different days and correlation between antimicrobial activity and a ratio of bee bread to $50 \%$ ethanol and between antimicrobial activity and total flavonoid content of bee bread extracts. Therefore, the aim of this research was to elaborate analytical procedure for measuring the total flavonoid content, establish precision parameters for the developed analytical procedure, determine antimicrobial properties of the extracts of bee bread collected in Ukraine in 2015 and 2016 and evaluate correlations of antimicrobial activity with a ratio of bee bread to $50 \%$ ethanol and the total flavonoid content. The parameters of the precision include repeatability (RSD for measurements in one day) and intermediate precision (a variation in a marker content in different days).

This study is expected to widen the existing information on the characteristic of the analytical procedure of bee bread extracts total flavonoid content determination, their antimicrobial activity and to assist in more focused future analytical and technological research aiming to develop bee bread extract for different applications in food and pharmaceutical industries.

\section{MATERIALS AND METHODS}

\section{Sample preparation}

Seven bee bread samples were used in this study. These samples were removed from hive cells, dried at a temperature of $36-38^{\circ} \mathrm{C}$ (with exception of bee bread of sample 3), packed in polypropylene bags and stored in a refrigerator at a temperature of $2-8^{\circ} \mathrm{C}$ before extracts were prepared. Bee bread of sample 3 was not dried before packaging. The samples were collected in Ukraine during the summer of 2015 and 2016. The hives were located in Vradivka of Mykolajiv region and Poltava region. Bee bread granules were put in containers, $50 \%(\mathrm{~m} / \mathrm{m})$ ethanol was added in necessary volume and extraction (maceration) was carried out at a temperature of 15 $-25^{\circ} \mathrm{C}$ with periodic stirring for 7-21 days at a ratio of raw material to the solvent of 1 to 20,1 to 10 and 1 to 5 . Then extracts were filtered through filter paper. $50 \%$ ethanol was selected as a solvent that extracts both hydrophilic (vitamin $\mathrm{C}$ and phenolic acids) and hydrophobic (flavonoids) biologically active substances of bee bread (7). Before carrying out analytical procedures extracts were additionally filtered through the filter with the pore size of 0.45 microns if necessary. Table 1 presents information about the tested bee bread samples and their extracts. 
Table 1. Characteristic of bee bread samples and extracts.

\begin{tabular}{|c|c|c|c|c|c|}
\hline $\begin{array}{l}\text { Number } \\
\text { of extract }\end{array}$ & $\begin{array}{l}\text { Number of bee } \\
\text { bread sample }\end{array}$ & $\begin{array}{c}\text { Date of bee bread } \\
\text { collection }\end{array}$ & $\begin{array}{c}\text { Ratio of bee bread } \\
\text { to } 50 \% \text { ethanol }\end{array}$ & $\begin{array}{c}\text { Dates of bee } \\
\text { bread maceration }\end{array}$ & $\begin{array}{c}\text { Time of bee } \\
\text { bread maceration }\end{array}$ \\
\hline 1 & 1 & 15.07 .2015 & $10.0 \mathrm{~g}$ to $100 \mathrm{~mL}$ & $19.04 .2016-10.05 .2016$ & 21 day \\
\hline 2 & 2 & 30.05 .2015 & $10.0 \mathrm{~g}$ to $100 \mathrm{~mL}$ & $29.11 .2016-06.12 .2016$ & 7 days \\
\hline 3 & 3 & June 2015 & $10.0 \mathrm{~g}$ to $100 \mathrm{~mL}$ & $19.04 .2016-10.05 .2016$ & 21 day \\
\hline 4 & 4 & 02.08 .2015 & $20.0 \mathrm{~g}$ to $100 \mathrm{~mL}$ & $10.05 .2016-27.05 .2016$ & 17 days \\
\hline 5 & 1 & 15.07 .2015 & $20.0 \mathrm{~g}$ to $100 \mathrm{~mL}$ & $10.05 .2016-27.05 .2016$ & $-/ /-$ \\
\hline 6 & 3 & June 2015 & $20.0 \mathrm{~g}$ to $100 \mathrm{~mL}$ & $10.05 .2016-27.05 .2016$ & $-/ /-$ \\
\hline 7 & 5 & 10.06 .2016 & $2.5 \mathrm{~g}$ to $50 \mathrm{~mL}$ & $28.09 .2016-07.10 .2016$ & 9 days \\
\hline 8 & 5 & 10.06.2016 & $2.5 \mathrm{~g}$ to $50 \mathrm{ml}$ & $28.09 .2016-07.10 .2016$ & $-/ /-$ \\
\hline 9 & 5 & 10.06.2016 & $2.5 \mathrm{~g}$ to $50 \mathrm{~mL}$ & $28.09 .2016-07.10 .2016$ & $-/ /-$ \\
\hline 10 & 6 & 23.05 .2016 & $2.5 \mathrm{~g}$ to $50 \mathrm{~mL}$ & $28.09 .2016-07.10 .2016$ & $-/ /-$ \\
\hline 11 & 6 & 23.05 .2016 & $2.5 \mathrm{~g}$ to $50 \mathrm{~mL}$ & $28.09 .2016-07.10 .2016$ & $-/ /-$ \\
\hline 12 & 1 & 15.07 .2015 & $5.0 \mathrm{~g}$ to $100 \mathrm{~mL}$ & $29.11 .2016-06.12 .2016$ & 7 days \\
\hline 13 & 7 & 22.06.2016 & $5.0 \mathrm{~g}$ to $100 \mathrm{~mL}$ & $29.11 .2016-06.12 .2016$ & $-/ /-$ \\
\hline 14 & 7 & 22.06 .2016 & $10.0 \mathrm{~g}$ to $100 \mathrm{~mL}$ & $29.11 .2016-06.12 .2016$ & $-/ /-$ \\
\hline 15 & 3 & June 2015 & $20.2 \mathrm{~g}$ to $200 \mathrm{~mL}$ & $29.11 .2016-06.12 .2016$ & $-/ /-$ \\
\hline 16 & 3 & June 2015 & $20.1 \mathrm{~g}$ to $100 \mathrm{~mL}$ & $29.11 .2016-06.12 .2016$ & $-/ /-$ \\
\hline
\end{tabular}

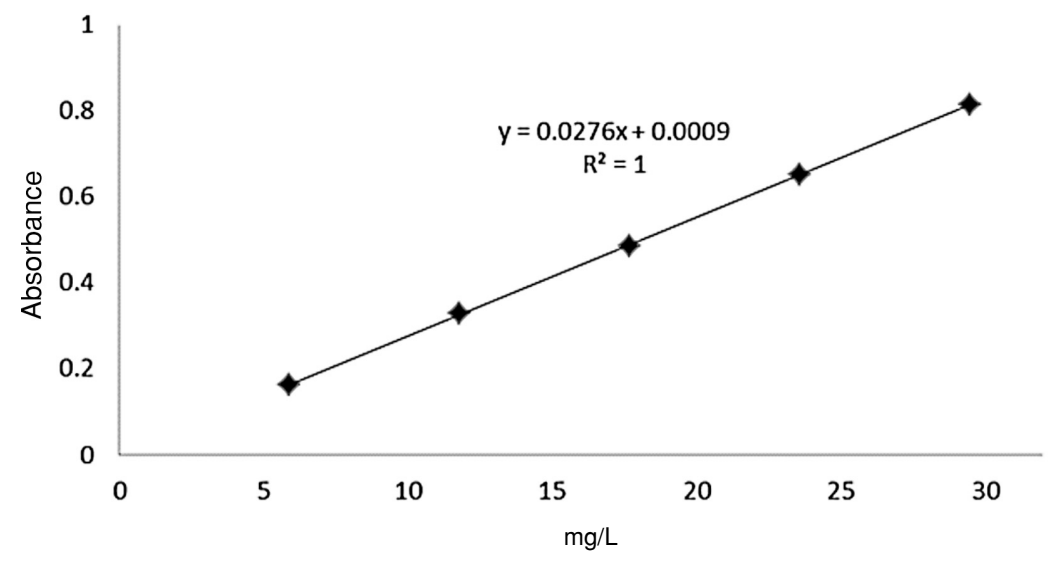

Figure 1. Calibration curve of quercetine dihydrate

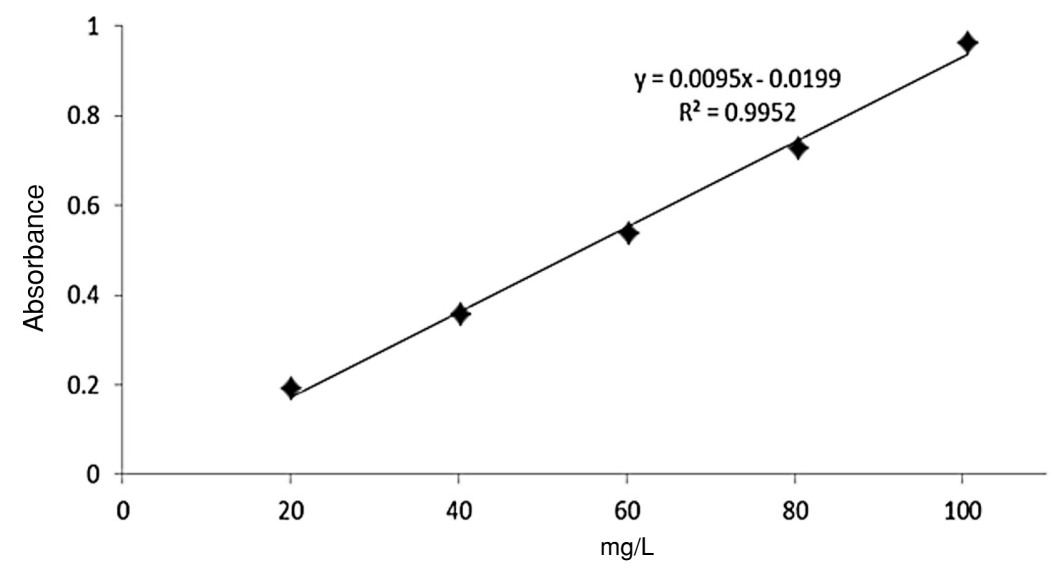

Figure 2. Calibration curve of rutin trihydrate 


\section{Total flavonoid content}

The total flavonoid content was determined using the slightly modified procedure of differential spectrometry provided by Chang C.C. et al. (17),
Meda A. et al. (18), Zhang et al. (19) and Ordonez et al. (20) using calibration curves of quercetin dihydrate and rutin trihydrate (Figs. 1 and 2, respectively). The slight modifications referred to either a wave-

Table 2. Total flavonoid content of the bee bread extracts with repeatability and intermediate precision.

\begin{tabular}{|c|c|c|c|c|}
\hline \multirow[b]{2}{*}{ Sample } & \multicolumn{2}{|c|}{ The total flavonoid content with reference to } & \multirow[b]{2}{*}{$\begin{array}{l}\text { Reaction time, } \\
\text { min }\end{array}$} & \multirow[b]{2}{*}{$\begin{array}{l}\text { Comment on the change in the mean } \\
\text { total flavonoid content }\end{array}$} \\
\hline & $\begin{array}{c}\text { Quercetin, } \\
\text { mean }(\mathrm{mg} / \mathrm{L}) \pm \mathrm{RSD}\end{array}$ & $\begin{array}{c}\text { Rutin, } \\
\text { mean }(\mathrm{mg} / \mathrm{L}) \pm \mathrm{RSD}\end{array}$ & & \\
\hline 1 & $107.2 \pm 1.91 \%$ & $340.3 \pm 7.70 \%$ & 85 & - \\
\hline 2 & $74.8 \pm 10.00 \%$ & $243.6 \pm 17.43 \%$ & 75 & - \\
\hline \multirow{2}{*}{3} & $8.3 \pm 6.24 \%$ & $28.8 \pm 19.33 \%$ & 75 & \multirow{2}{*}{$\begin{array}{l}\text { There is an increase in the mean content by } \\
3.6 \% \text { and } 2.8 \% \text { with reference to quercetin } \\
\text { and rutine, respectively }\end{array}$} \\
\hline & $8.6 \pm 3.11 \%$ & $29.6 \pm 16.29 \%$ & 118 & \\
\hline \multirow[t]{2}{*}{4} & $158.4 \pm 4.32 \%$ & $493.1 \pm 8.22 \%$ & 84 & \multirow{2}{*}{$\begin{array}{l}\text { There is an increase in the mean content by } \\
1.8 \% \text { with reference to quercetin and rutine }\end{array}$} \\
\hline & $161.3 \pm 3.65 \%$ & $501.8 \pm 7.51 \%$ & 116 & \\
\hline 5 & $165.5 \pm 3.82 \%$ & $514.4 \pm 7.58 \%$ & 75 & - \\
\hline \multirow{2}{*}{6} & $14.0 \pm 6.54 \%$ & $45.8 \pm 14.75 \%$ & 70 & \multirow{2}{*}{$\begin{array}{l}\text { There is an increase in the mean content by } \\
3.6 \% \text { and } 3.1 \% \text { with reference to quercetin } \\
\text { and rutine, respectively }\end{array}$} \\
\hline & $14.5 \pm 3.52 \%$ & $47.2 \pm 11.74 \%$ & 82 & \\
\hline $7 *$ & $135.2 \pm 9.4 \%$ & - & 75 & - \\
\hline \multirow{3}{*}{$8 *$} & $125.7 \pm 11.64 \%$ & - & 75 & \multirow{3}{*}{$\begin{array}{l}\text { There is an increase in the mean } \\
\text { content by } 2.3 \% \text { with reference } \\
\text { to quercetin }\end{array}$} \\
\hline & & In 6 months & & \\
\hline & $128.6 \pm 4.45 \%$ & $404.1 \pm 9.21 \%$ & 75 & \\
\hline $9 *$ & $136.1 \pm 10.39 \%$ & - & 77 & - \\
\hline \multirow{4}{*}{10} & $118.5 \pm 0.23 \%$ & $374.2 \pm 5.59 \%$ & 75 & \multirow{2}{*}{$\begin{array}{l}\text { There is a decrease in the mean content by } \\
0.3 \% \text { with reference to quercetin and rutine }\end{array}$} \\
\hline & $118.2 \pm 1.82 \%$ & $373.2 \pm 7.11 \%$ & 120 & \\
\hline & \multicolumn{3}{|c|}{ In 3 days } & \\
\hline & $107.5 \pm 1.47 \%$ & $341.3 \pm 7.27 \%$ & 82 & $\begin{array}{l}\text { There is a decrease in the mean content by } \\
9.3 \% \text { and } 8.8 \% \text { with reference to quercetin } \\
\text { and rutine, respectively, from the initial } \\
\text { values }\end{array}$ \\
\hline \multirow{3}{*}{11} & $110.4 \pm 2.03 \%$ & $350.0 \pm 7.66 \%$ & 75 & \multirow{3}{*}{$\begin{array}{l}\text { There is a decrease in the mean content by } \\
4.1 \% \text { and } 3.9 \% \text { with reference to quercetin } \\
\text { and rutine, respectively, from the initial } \\
\text { values }\end{array}$} \\
\hline & & In 7 days & & \\
\hline & $105.9 \pm 0.80 \%$ & $336.4 \pm 6.73 \%$ & 85 & \\
\hline \multirow{3}{*}{12} & $78.1 \pm 2.50 \%$ & $253.3 \pm 10.24 \%$ & 85 & \multirow{3}{*}{$\begin{array}{l}\text { There is an increase in the mean content by } \\
9.9 \% \text { and } 9.2 \% \text { with reference to quercetin } \\
\text { and rutine, respectively, from the initial } \\
\text { values }\end{array}$} \\
\hline & & In 1 day & & \\
\hline & $85.8 \pm 4.01 \%$ & $276.5 \pm 10.99 \%$ & 84 & \\
\hline \multirow{4}{*}{13} & $128.2 \pm 4.74 \%$ & $403.2 \pm 9.49 \%$ & 83 & \multirow{4}{*}{$\begin{array}{l}\text { 1. There is a decrease in the mean content } \\
\text { by } 10.6 \% \text { and } 10.1 \% \text { with reference to } \\
\text { quercetin and rutine, respectively, at } 83 \text { and } \\
85 \text { min of different days from the initial } \\
\text { values; } \\
2 \text {. There is an increase in the mean content } \\
\text { by } 3.8 \% \text { and } 3.6 \% \text { with reference to } \\
\text { quercetin and rutine, respectively, at } 85 \mathrm{~min} \\
\text { from the initial values at } 77 \text { min }\end{array}$} \\
\hline & \multicolumn{3}{|c|}{ In 2 days } & \\
\hline & $110.4 \pm 1.82 \%$ & $350.0 \pm 7.46 \%$ & 77 & \\
\hline & $114.6 \pm 1.95 \%$ & $362.6 \pm 7.39 \%$ & 85 & \\
\hline 14 & $195.3 \pm 1.35 \%$ & $603.3 \pm 4.64 \%$ & 75 & - \\
\hline 15 & $9.5 \pm 5.29 \%$ & $32.3 \pm 17.08 \%$ & 75 & - \\
\hline 16 & $14.7 \pm 11.08 \%$ & $48.0 \pm 18.54 \%$ & 85 & - \\
\hline
\end{tabular}

\footnotetext{
*- determination was carried out only for quercetin
} 


\begin{tabular}{|c|c|c|c|c|c|c|c|c|c|c|c|c|c|c|c|c|c|c|c|c|c|c|}
\hline \multirow{2}{*}{\multicolumn{2}{|c|}{$\begin{array}{l}\bar{D} \\
\overline{0} \\
\frac{0}{0} \\
\frac{0}{0} \\
\overline{0}\end{array}$}} & \multicolumn{2}{|c|}{ 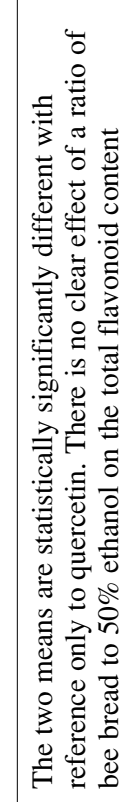 } & \multicolumn{2}{|c|}{ 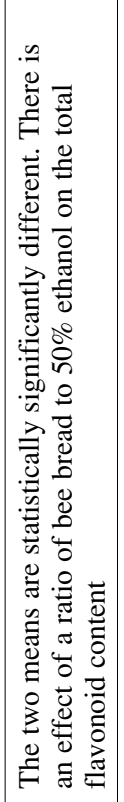 } & \multicolumn{2}{|c|}{ 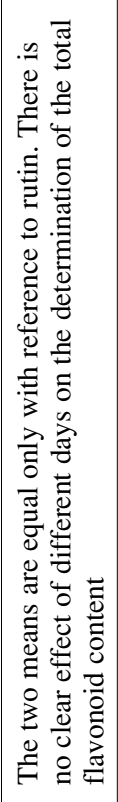 } & \multicolumn{2}{|c|}{ 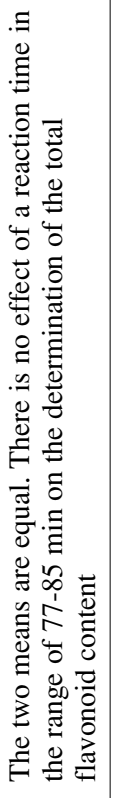 } & \multicolumn{2}{|c|}{ 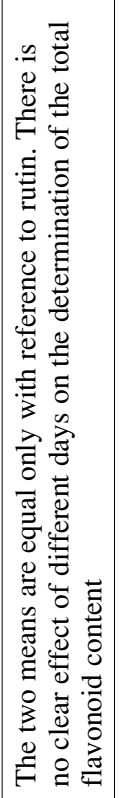 } & 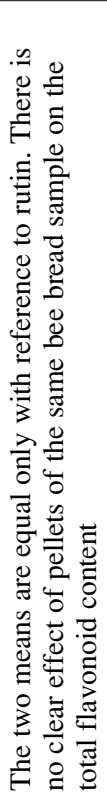 & \multicolumn{2}{|c|}{ 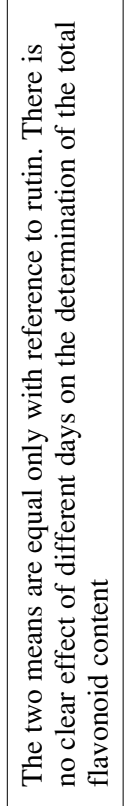 } & \multicolumn{2}{|c|}{ 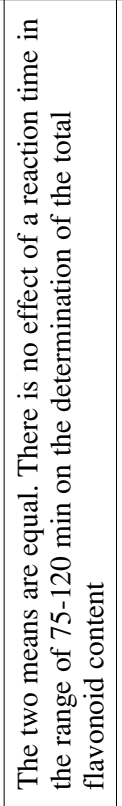 } & \multicolumn{2}{|c|}{ 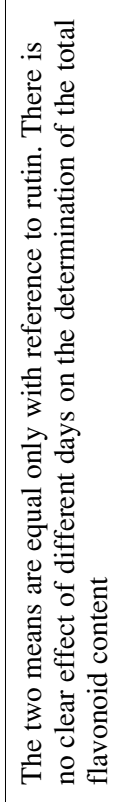 } & & & \multicolumn{2}{|c|}{ 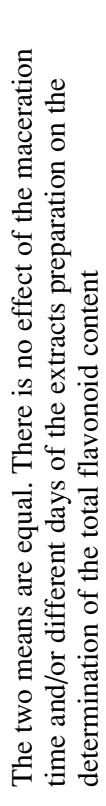 } \\
\hline & & \multirow{2}{*}{ 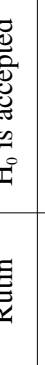 } & \multicolumn{2}{|c|}{ 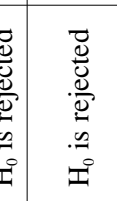 } & \multirow{2}{*}{\multicolumn{2}{|c|}{ 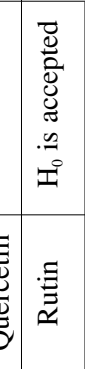 }} & 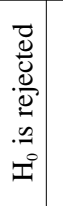 & 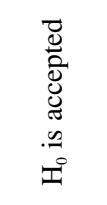 & & \multirow{2}{*}{\multicolumn{2}{|c|}{ 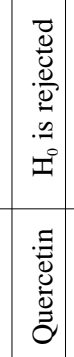 }} & \multirow{2}{*}{ 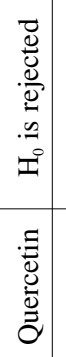 } & \multirow{2}{*}{ 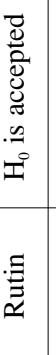 } & 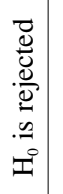 & \multicolumn{2}{|c|}{ 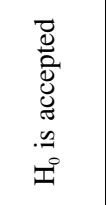 } & 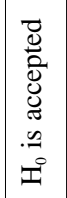 & 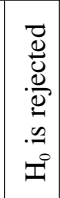 & \multicolumn{2}{|c|}{ 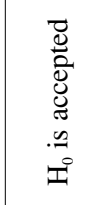 } & \multicolumn{2}{|c|}{ 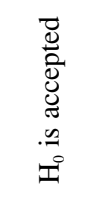 } \\
\hline 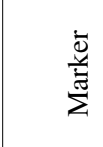 & 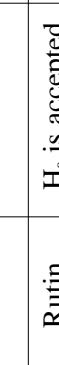 & & 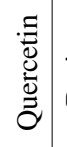 & 䒠 & & & 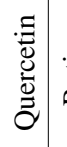 & 夆 & & & & & & 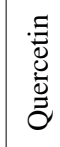 & 志 & 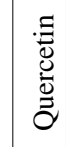 & 害 & $\mid \begin{array}{l}: \\
\overline{0} \\
\overline{0} \\
\tilde{\Xi} \\
\tilde{0}\end{array}$ & & & & \\
\hline & $\begin{array}{l}\hat{6} \\
\text { în }\end{array}$ & \begin{tabular}{l}
$\infty$ \\
\multirow{i}{1}{} \\
$\dot{r}$
\end{tabular} & $\stackrel{\vec{r}}{r}$ & $\begin{array}{l}n \\
\stackrel{n}{s} \\
=\end{array}$ & $\stackrel{g}{-}$ & $\begin{array}{c}\stackrel{1}{*} \\
\stackrel{+}{+}\end{array}$ & $\left.\begin{array}{ll}\infty \\
\infty \\
i \\
i \\
i\end{array}\right]$ & 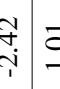 & $\stackrel{9}{9}$ & ?ִ & तु & $\stackrel{?}{\stackrel{9}{i}}$ & 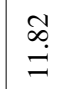 & $\stackrel{0}{0}$ & 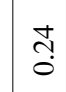 & $\begin{array}{l}5 \\
0\end{array}$ & 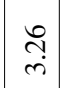 & $\stackrel{?}{0}$ & $0^{\infty}$ & $0^{\circ}$ & \\
\hline \multicolumn{2}{|l|}{$\tilde{n}=$} & 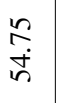 & $\stackrel{\text { q }}{-}$ & $\underset{\stackrel{+}{I}}{\Xi}$ & $\begin{array}{c}\bar{\sigma} \\
\vec{\sim} \\
\vdots\end{array}$ & 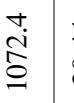 & $\begin{array}{l} \pm \\
\stackrel{d}{\Delta}\end{array}$ & 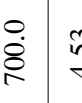 & 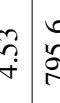 & $\stackrel{\infty}{\infty}$ & \begin{tabular}{c}
0 \\
\hdashline \\
\end{tabular} & ֶn & 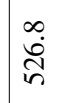 & ते & $\frac{\hat{\lambda}}{\vec{\lambda}}$ & $\vec{m}$ & $\begin{array}{l}+ \\
i \\
0 \\
0\end{array}$ & $\begin{array}{l}\widehat{\infty} \\
\stackrel{i}{i}\end{array}$ & & $\dot{b}$ & نُ & \\
\hline \multicolumn{2}{|l|}{$\begin{array}{l}1 x^{\mid} \\
1 \\
1 x^{-1}\end{array}$} & in & $\stackrel{\sim}{n}$ & $\overline{\stackrel{8}{0}}$ & $\overrightarrow{\dot{0}}$ & $\begin{array}{r}n \\
\\
n\end{array}$ & $\stackrel{\infty}{=}$ & İ & $\begin{array}{r}\tilde{y} \\
\dot{y}\end{array}$ & $i$ & $\stackrel{\substack{I \\
i}}{i}$ & $\bar{\infty}$ & $\begin{array}{l}\text { ते } \\
\text { ले }\end{array}$ & $=$ & - & ?3 & $M$ & $\stackrel{n}{+}$ & $\begin{array}{l}0 \\
\sigma\end{array}$ & 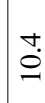 & $\dot{0}$ & \\
\hline \multirow{2}{*}{ 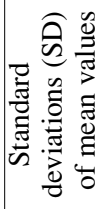 } & คิ & $\begin{array}{l}\stackrel{\phi_{0}}{\infty} \\
\infty\end{array}$ & $\stackrel{\leftrightarrow}{-}$ & $\begin{array}{l}\widetilde{d} \\
\text { d. }\end{array}$ & 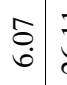 & $\vec{\nabla}$ & $\overrightarrow{\vec{i}}$ & 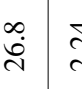 & \begin{tabular}{c|c}
$\vec{i}$ \\
$\vec{i}$
\end{tabular} & $\stackrel{\sharp}{\sharp}$ & 寸 & $\begin{array}{c}\text { i } \\
\text { i }\end{array}$ & さ̇ & $\stackrel{\infty}{n}$ & i & $\stackrel{\sim}{i}$ & \begin{tabular}{|l}
\multirow{0}{0}{} \\
ì
\end{tabular} & $\begin{array}{l}n \\
\infty \\
0 \\
0\end{array}$ & $\begin{array}{l}\text { है } \\
\text { త্ }\end{array}$ & $\cong$ & in & \\
\hline & $\hat{n}$ & $\begin{array}{l}\tilde{n} \\
\text { nू }\end{array}$ & $\ddot{0}$ & 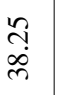 & $\begin{array}{l}\tilde{b} \\
\dot{i} \\
i\end{array}$ & ָे & s. & $\begin{array}{l}\bar{\alpha} \\
\overrightarrow{0}\end{array}$ & \begin{tabular}{c|c}
$\tilde{c}_{i}$ & 0 \\
$y$
\end{tabular} & 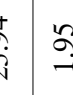 & ¿ُ & $\begin{array}{l}\text { तิ } \\
\text { o }\end{array}$ & సి & 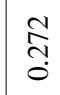 & ते & స઼ & i & 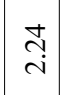 & $\overrightarrow{\mathrm{I}}$ & $\cong$ & $\infty$ & \\
\hline \multirow{2}{*}{ 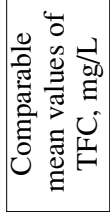 } & & $\underset{\infty}{\dot{\sigma}}$ & $\stackrel{\check{\dot{I}}}{\mathrm{I}}$ & జి. & 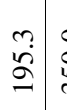 & ஜृ. & $\stackrel{⿱}{\ominus}$ & $=$ & 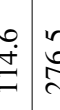 & $\infty$ & $\dot{c} \dot{c}$ & $\stackrel{+}{\varrho}$ & $\stackrel{m}{\vec{f}}$ & 管 & 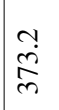 & $\begin{array}{l}\stackrel{n}{s} \\
\stackrel{\sigma}{\sigma}\end{array}$ & ల్ల & $\dot{\tilde{\delta}}$ & 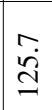 & $\mathcal{E}$ & F & \\
\hline & $|x|$ & ले & $n$ & $\oint$ & 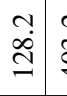 & & 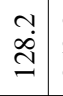 & $\begin{array}{l}\stackrel{8}{\circ} \\
\text { ले }\end{array}$ & $\stackrel{t}{\stackrel{t}{*}}$ & $\stackrel{\infty}{\sim}$ & $e^{\circ}$ & $\stackrel{\infty}{=}$ & & $\begin{array}{l}n \\
= \\
=\end{array}$ & $\stackrel{d}{d}$ & $\begin{array}{l}\stackrel{n}{\infty} \\
= \\
=\end{array}$ & हn & $\stackrel{t}{\circ}$ & 2 & 2 & ó & \\
\hline \multirow{2}{*}{ 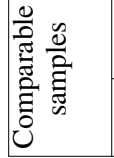 } & & $\approx$ & & & & & & & & & & & & & & & & & $\infty$ & $\infty$ & & \\
\hline & - & - & & - & & & & - & & & & $=$ & & & & 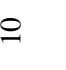 & & & & a & & \\
\hline
\end{tabular}




\begin{tabular}{|c|c|c|c|c|c|c|c|c|c|c|c|c|c|}
\hline \multicolumn{2}{|c|}{$\begin{array}{l}n \\
\tilde{0} \\
0 \\
\overline{0} \\
\overline{0} \\
0 \\
0\end{array}$} & \multicolumn{2}{|c|}{ 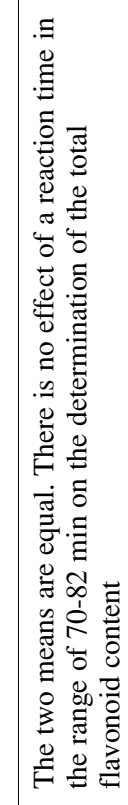 } & \multicolumn{2}{|c|}{ 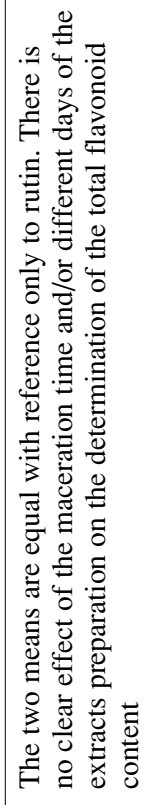 } & \multicolumn{2}{|c|}{ 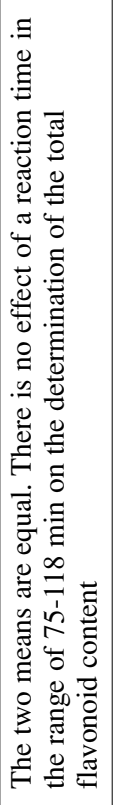 } & \multicolumn{2}{|c|}{ 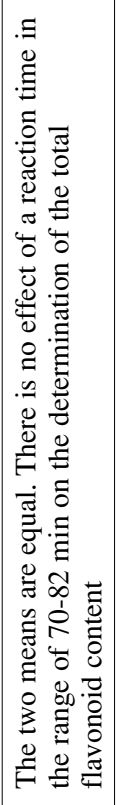 } & \multicolumn{4}{|c|}{ 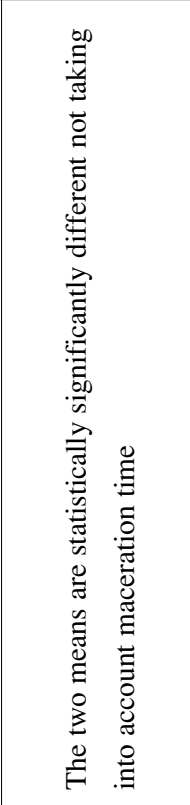 } \\
\hline \multicolumn{2}{|l|}{$\begin{array}{l}\bar{\Xi} \\
\overline{0} \\
\overline{0} \\
\overline{0} \\
\overline{0}\end{array}$} & \multicolumn{2}{|c|}{ 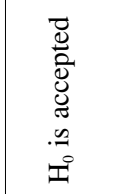 } & 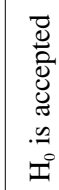 & 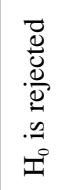 & \multicolumn{2}{|c|}{ 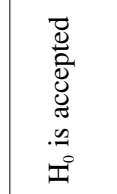 } & \multicolumn{2}{|c|}{ 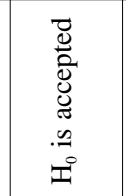 } & \multicolumn{2}{|c|}{ 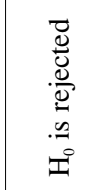 } & \multicolumn{2}{|c|}{ 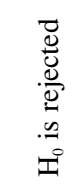 } \\
\hline \multicolumn{2}{|l|}{ 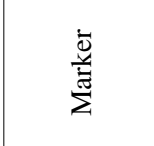 } & 声 & 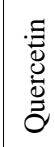 & $\stackrel{\Xi}{\widetilde{2}}$ & 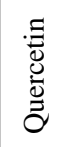 & 声 & 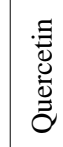 & 声 & 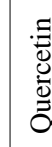 & 吾 & 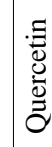 & 咅 & $\begin{array}{l}\text { 志 } \\
\frac{0}{2} \\
\bar{\Xi} \\
\end{array}$ \\
\hline \multicolumn{2}{|l|}{-} & 文 & $\begin{array}{l}\overrightarrow{0} \\
\stackrel{0}{0}\end{array}$ & $\stackrel{ }{\hat{\sigma}}$ & $\begin{array}{l}\infty \\
\infty \\
i\end{array}$ & $\frac{\partial}{0}$ & $\bar{\sigma}$ & 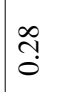 & $\stackrel{\infty}{0}$ & $\begin{array}{l}\stackrel{8}{ } \\
\dot{+}\end{array}$ & 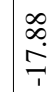 & f̊. & $\begin{array}{l}\tilde{\lambda} \\
\ddot{n}\end{array}$ \\
\hline \multicolumn{2}{|l|}{ in } & $\begin{array}{l}\infty \\
\stackrel{\infty}{n} \\
\hat{m}\end{array}$ & $\begin{array}{l}\text { : } \\
\text { : }\end{array}$ & 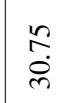 & ֻ̊ & $\stackrel{\sim}{\stackrel{\sim}{\sim}}$ & $\stackrel{7}{0}$ & $\begin{array}{l}\stackrel{1}{1} \\
\infty \\
\infty \\
m\end{array}$ & in & $\stackrel{\infty}{\stackrel{0}{0}}$ & $\begin{array}{l}\infty \\
\stackrel{m}{m}\end{array}$ & & $\stackrel{\infty}{\infty}$ \\
\hline \multicolumn{2}{|l|}{$\begin{array}{c}|x| \\
1 \\
1 \\
\mid x\end{array}$} & $\stackrel{+}{\rightarrow}$ & ?? & $\ddot{n}$ & $\stackrel{\sim}{\simeq}$ & $\stackrel{\infty}{0}$ & ?3 & $\stackrel{\infty}{\stackrel{\infty}{\sim}}$ & in & $\underset{\substack{\infty \\
\text { i. }}}{.}$ & İ & $\begin{array}{l}\stackrel{9}{+} \\
\stackrel{+}{I}\end{array}$ & 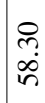 \\
\hline \multirow{2}{*}{ 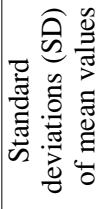 } & की & 鸪 & $\bar{n}$ & 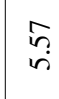 & กี & 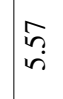 & $\tilde{n}$ & $\stackrel{0}{6}$ & $\tilde{\delta}$ & तु & $\underset{i}{\stackrel{i}{i}}$ & $\begin{array}{l}\text { त̦ } \\
\text { }\end{array}$ & $\underset{i}{\stackrel{i}{i}}$ \\
\hline & की & $\stackrel{0}{\stackrel{0}{6}}$ & $\delta$ & $\begin{array}{l}\tilde{n} \\
\text { ñ }\end{array}$ & n? & $\begin{array}{l}\mathcal{C} \\
\stackrel{+}{+}\end{array}$ & సิ & $\begin{array}{l}\stackrel{+}{n} \\
\dot{n}\end{array}$ & $\sqrt{n}$ & $\begin{array}{l}\text { ते } \\
\text { பे }\end{array}$ & $\stackrel{\leftrightarrow}{\Omega}$ & & तె \\
\hline \multirow{2}{*}{ 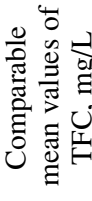 } & 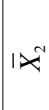 & $\stackrel{\stackrel{\sim}{q}}{\underset{f}{q}}$ & $\stackrel{n}{ \pm}$ & $\begin{array}{l}\infty \\
\dot{\sim} \\
\dot{\sim}\end{array}$ & $\infty$ & $\begin{array}{l}\infty \\
\dot{i} \\
\dot{v}\end{array}$ & $\stackrel{m}{\infty}$ & 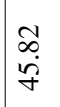 & $\stackrel{\circ}{ \pm}$ & 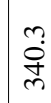 & 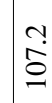 & & $\stackrel{\overbrace{}}{\stackrel{\overbrace{}}{\sigma}}$ \\
\hline & $\mid x-1$ & $\begin{array}{l}\infty \\
\dot{y} \\
\dot{f}\end{array}$ & $\stackrel{\circ}{\dot{I}}$ & in & $n$ & $\begin{array}{l}\text { तें } \\
\dot{\hat{\lambda}}\end{array}$ & $\stackrel{\circ}{\infty}$ & 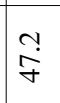 & $\stackrel{n}{ \pm}$ & $\tilde{\mathrm{n}}$ & 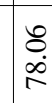 & & 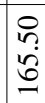 \\
\hline \multirow{2}{*}{ 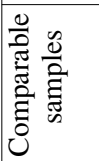 } & $\alpha$ & \multicolumn{2}{|c|}{0} & \multicolumn{2}{|c|}{. } & \multicolumn{2}{|c|}{$m$} & \multicolumn{2}{|c|}{ 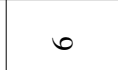 } & & \multicolumn{2}{|c|}{-} \\
\hline & - & \multicolumn{2}{|c|}{0} & & \multicolumn{2}{|c|}{$m$} & \multicolumn{2}{|c|}{0} & & $\simeq$ & & $n$ \\
\hline
\end{tabular}


length for measuring, reaction time or volume of sample for analysis, etc.

The calibration curves of quercetin dihydrate and rutin trihydrate were plotted in the range of concentrations of 2.08 to $31.2 \mathrm{mg} / \mathrm{L}$ and 20.12 to 100.6 $\mathrm{mg} / \mathrm{L}$, respectively. $1.0 \mathrm{~mL}$ of the obtained solutions of quercetin dihydrate or rutin trihydrate was mixed with $1.0 \mathrm{~mL}$ of $2 \%$ aluminum chloride hexahydrate in $50 \%$ ethanol. The amount of $2 \%$ aluminum chloride hexahydrate in $50 \%$ ethanol was substituted by the same volume of $50 \%$ ethanol in the blank for each solution of quercetin dihydrate or rutin trihydrate, respectively. The mixtures were vortexed. After incubation at room temperature for $80 \pm 5 \mathrm{~min}$, the absorbance of the reaction mixtures was measured at $425 \mathrm{~nm}$ for quercetin dihydrate and $410 \mathrm{~nm}$ for rutin trihydrate (19).

In most cases measurements were carried out at 404-430 nm with using different flavonols (quercetin, rutin, quercetrin, galangin) as well as flavan-3-ol catechin as markers (21). For studies of natural products with quercetin as marker authors perform measurements at one of the following wavelengths $415 \mathrm{~nm}(2,17,18,22,23), 420 \mathrm{~nm}(20), 430$ $\mathrm{nm}(21)$ and even $510 \mathrm{~nm}$ in the presence of $\mathrm{NaNO}_{2}$ (3) and with rutin at one of the following wavelengths: $510 \mathrm{~nm}$ (16) and $410 \mathrm{~nm}$ (19). In previous studies, it was confirmed that quercetin dihydrate and rutin trihydrate had maximum absorption at wavelengths of $425 \pm 2 \mathrm{~nm}$ and $411 \pm 1 \mathrm{~nm}$, respectively. Taking into account literature and own data, such wavelengths of 425 and $410 \mathrm{~nm}$ were used for the measurements. The mean of three readings was used for each concentration of the active marker.

In a like manner, the certain volume of an extract of bee bread was diluted with $50 \%$ ethanol up to $1.0 \mathrm{~mL}$ and was mixed with 1.0 of $2.0 \%$ solution of aluminum chloride hexahydrate in 50\% ethanol. As a rule, $0.1 \mathrm{~mL}$ of an extract was taken with the exception of $0.5 \mathrm{~mL}$ for samples 3 and 6 with lower total flavonoid content. The amount of $2 \%$ aluminum chloride hexahydrate in $50 \%$ ethanol was substituted by the same volume of $50 \%$ ethanol in the blank. The mixture was vortexed and incubated for 75-85 min. Some mixtures were incubated up to $120 \mathrm{~min}$ at room temperature for studying time's influence on the absorbance of the reaction mixtures. $80 \pm 5 \mathrm{~min}$ was chosen a compromise reaction time for assay of the total flavonoid content.

Then the absorbances of the reaction mixtures were measured at $410 \mathrm{~nm}$. The test was carried out for each bee bread extract in triplicate.

In previous unpublished studies, it was established that the maximum absorption of the reaction mixtures of the bee bread extracts varied in the range of 403 to $415 \mathrm{~nm}$. Therefore, a wavelength of $410 \mathrm{~nm}$ was chosen as a compromise wavelength for assay of the total flavonoid content.

The appropriate equations for the calibration curves of quercetin dihydrate and rutin trihydrate in $\mathrm{mg} / \mathrm{L}\left(\mathrm{Y}_{1}=0.0276 \cdot \mathrm{X}_{1}+0.0009\right.$ and $\mathrm{Y}_{2}=0.0095 \cdot \mathrm{X}_{2}$ -0.0199 , respectively), necessary recalculations for converting quercetin dihydrate into quercetin, rutin trihydrate into rutin and volume of an extract $(0.1$ or $0.5 \mathrm{~mL}$ ) taken for the analytical procedure were used for calculations of the total flavonoid content in the tested extracts. For samples 7, 8 and 9 the following equation was used for only quercetin dihydrate $\left(\mathrm{Y}_{3}=\right.$ $\left.0.0299 \cdot X_{3}-0.0219, r=0.998\right)$. The mean of three readings was used and expressed as $\mathrm{mg}$ of quercetin or rutin equivalents per $1 \mathrm{~L}$ of an extract.

\section{Antimicrobial activity}

The ethanolic bee bread extracts were subjected to evaporation under reduced pressure at $40^{\circ} \mathrm{C}$ in order to remove ethanol (Stuart RE300DB rotary evaporator, Bibby scientific limited, UK, vacuum pump KNF N838.1.2KT.45.18, KNF, Germany). For the antimicrobial assay, the dried bee bread extracts were dissolved in dimethyl sulfoxide (DMSO) (Penta, Czech Republic).

\section{Microorganisms}

Fifteen strains of microorganisms were tested in this study, including three Gram-negative bacteria (Haemophilus influenzae CCM 4456, Klebsiella pneumoniae CCM 2318, Salmonella enterica subsp. enterica CCM 3807), three Gram-positive bacteria (Bacillus cereus CCM 2010, Clostridium perfringens CCM 4435, Staphylococcus aureus subsp. aureus CCM 4223), three yeasts (Candida albicans CCM 8186, Candida glabrata CCM 8270, Candida tropicalis CCM 8223) and six microscopic filamentous fungi (Aspergillus clavatus, Aspergillus flavus, Aspergillus versicolor, Penicillium expansum, Penicillium chrysogenum, and Penicillium griseofulvum). The tested bacterial and yeasts strains were collected from the Czech Collection of microorganisms and microscopic fungi from the Department of Microbiology of Faculty of biotechnology and food sciences of the Slovak University of Agriculture in Nitra. The bacterial strains were grown in MuellerHinton agar (MHA, Biolife, Italy) in plates at a temperature of $37^{\circ} \mathrm{C}$, whereas the yeasts and molds were grown in Sabouraud dextrose agar (SDA, Biolife, Italy) and Potato dextrose agar (PDA, Biolife, Italy), respectively, at a temperature of $28^{\circ} \mathrm{C}$. The stock cultures were maintained at a temperature of $4^{\circ} \mathrm{C}$. 


\section{Disc diffusion method}

Antimicrobial activity of each bee bread extract was determined by a disc diffusion method. Briefly, $100 \mu \mathrm{L}$ of the tested microorganisms were grown in $10 \mathrm{~mL}$ of fresh media until they reached a count of approximately $10^{5}$ cells per $\mathrm{mL}$. Then $100 \mu \mathrm{L}$ of the microbial suspension was spread onto Mueller Hinton agar plates. The extracts were tested using 6 $\mathrm{mm}$ sterilized filter paper discs. The diameters of the inhibition zones were measured in millimeters. All the measurements were round up to the closest whole millimeter. Each antimicrobial assay was performed at least three times. Blank discs were impregnated with Ampicillin (10 $\mu \mathrm{g} / \mathrm{disc}$, Oxoid, UK) for Grampositive bacteria and yeasts, Gentamicin $(10 \mu \mathrm{g} / \mathrm{disc}$, Oxoid, UK) for Gram-negative bacteria and griseofulvin $(25 \mu \mathrm{g} / \mathrm{disc}$, Oxoid, UK) for microscopic filamentous fungi and used as positive controls. The negative control was a $1 \%$ solution of DMSO.

\section{Statistical analysis}

All analyses for each sample were carried out in triplicate and the results were expressed as mean value \pm standard deviation (SD) or mean value \pm relative standard deviation (RSD).

Correlation coefficients (r) to determine relationships between values were calculated using MS Excel Software. Achim Buyul and Peter Tsefel's classification was employed to estimate found correlation coefficients ( $\mathrm{r}$ ) between two magnitudes: up to 0.2 - very weak, up to 0.5 - weak, up to 0.7 medium, up to 0.9 high and over 0.9 very high correlation (24).

Wayne's statistical analysis was employed for comparison of the total flavonoid content mean values obtained over a short interval of time, in different days, for the different ratio of bee bread to solvent, etc. Decision rule in all cases was: with $\alpha=$ 0.025 , critical values of $t *$ should be in the range of -2.78 to +2.78 . Hull hypothesis $\left(\mathrm{H}_{0}\right)$ was rejected if $\mathrm{t}^{*}<-2.78$ or $\mathrm{t}^{*}>+2.78(25)$.

\section{RESULTS AND DISCUSSION}

The total flavonoid content of the extracts prepared with the different ratio of bee bread to $50 \%$ ethanol showed values of $8.3 \mathrm{mg} / \mathrm{L} \pm 6.24 \%$ to 195.3 $\mathrm{mg} / \mathrm{L} \pm 1.35 \%$ with reference to quercetin and 28.8 $\mathrm{mg} / \mathrm{L} \pm 19.33 \%$ to $603.3 \mathrm{mg} / \mathrm{L} \pm 4.64 \%$ with reference to rutin. The highest total flavonoid content was observed for the extracts of bee bread with a ratio of bee bread to $50 \%$ ethanol as 1 to 5 and 1 to 10 .

Flavonoids including those of bee bread are considered important for their health-promoting activity and high nutritional value (3, 6, 23). They possess antioxidant and anti-inflammatory activities (23). They exceed vitamin $\mathrm{C}$ and vitamin $\mathrm{E}$ in terms of antioxidant activity because they have a stronger capacity to donate electrons (23). Standardization of some plant materials is recommended to be carried out based on the total flavonoid content (26).

The principle of aluminum chloride colorimetric method consists in that aluminum chloride mainly forms complexes with flavones, flavonols and chalcones. Aluminum chloride reacts with the C-4 keto group and either the $\mathrm{C}-3$ hydroxyl group of the ring $\mathrm{C}$ and/or $\mathrm{C}-5, \mathrm{C}-7$ hydroxyl group of the ring $\mathrm{A}$ and the $\mathrm{C}-3^{\prime}$ or $\mathrm{C}-4^{\prime}$ of the ring $\mathrm{B}$ hydroxyl groups $(17,27)$.

According to the Guideline on quality of herbal medicinal products/traditional herbal medicinal products selected markers for assay should be justified (28). The authors of the published papers with exception of Chang et al. did not explain why they selected the proper marker or wavelength for the evaluation of flavonoid content in natural samples $(2,3,16,17)$.

Baltrušaityte at al. identified kaempferol, chrysin and apigenin in bee bread (14). Our previous study confirms that the complexes of quercetin with aluminum chloride have the maximum absorption at $423.8-427.2 \mathrm{~nm}$ in differential spectra in the concentration range of 2.08 to $31.2 \mathrm{mg} / \mathrm{L}$ (29). Markiewicz-Żukowska et al. detected kaempferol and apigenin in bee bread (6) while Čeksteryte et al. identified aglycons kaempferol and quercetin and stated that flavonoids in the form of glycosides have not been found in bee bread (5). Sobral et al. found flavonol derivatives, mainly quercetin, kaempferol, myricetin, isorhamnetin and herbacetrin glycoside derivatives (30). Tavdidishvili et al. reported the presence of such three flavonoids as naringin, quercetin and rutin in Georgian bee bread samples using HPLC-UV-Vis (31). Ursan et al. stated about presence of flavonol glycosides mainly from quercetin, kaempferol, 8-methylherbacetin and 7-methylherbacetin, determined total flavonoid content using a calibration curve of quercetin and checked the linearity of detector response using rutin as a standard (32). Therefore, quercetin and rutin could be employed as active markers for determination of the total flavonoid content as they were stated as components of bee bread $(3,31)$.

The total flavonoid content with aspects of precision of the elaborated analytical procedure is presented in Table 2.

To our knowledge, there is no regulation for RSD for parallel measurements in analytical proce- 
dures of flavonoids sum determination. In our studies, the RSD for parallel measurements for the two calibration curves of quercetin dihydrate $\left(\mathrm{Y}_{1}=\right.$ $0.0276 \cdot \mathrm{X}_{1}+0.0009$ and $\left.\mathrm{Y}_{3}=0.0299 \cdot \mathrm{X}_{1}-0.0219\right)$ and that of rutin trihydrate were in the range of $0.51 \%$ to $4.36 \%, 6.32$ to $9.39 \%$ and $5.02 \%$ to $19.91 \%$, respectively. The RSD for parallel measurements for the extracts with reference to quercetin dihydrate was in the range of $0.23 \%$ to $11.64 \%$ and $4.64 \% 19.33 \%$ with reference to rutin trihydrate. The difference between the two calibration curves of quercetin dihydrate could be explained by the different two intercepts, respectively, 0.0009 and 0.0219. The RSD of the extracts are similar to RSD obtained in other studies $(16,18,22)$. RSD ranged from 2.19 to $4.8 \%$ and 0 to $41.18 \%$ in studies related to determination of the total flavonoid content using a standard curve of quercetin in walnut green husks and honey $(18,22)$. RSD was $6.2 \%$ in studies related to determination of the total flavonoid content using a standard curve of rutin in root extract of Arisaema jacquemontii Blume (16).

According to the results in Table 2, the total flavonoid content depended on a ratio of bee bread to $50 \%$ ethanol $(\mathrm{m} / \mathrm{V})$ and technology of bee bread obtaining. Conspicuous results were obtained for different ratios of bee bread to $50 \%$ ethanol. The larger was the content of bee bread in $50 \%$ ethanol, the higher was the total flavonoid content. The increase of bee bread in the solvent in 2 times yields elevation of the total flavonoid content in approximately 1.5 times. Such an increase was observed for extract number 16 compared to number 15 and extract number 14 compared to number 13 .

The obtained results also demonstrated that the same sample of bee bread has approximately the same total flavonoid content in the extracts that could be explained by forming pellets of bee bread from pollen collected from the same flowers at the same time. For instance, extracts 7,8 and 9 contained approximately the same total flavonoid content at the same ratio of bee bread to $50 \%$ ethanol.

These studies also show that 7 days of maceration is enough for flavonoid extraction. For instance, the total flavonoid content of extracts number 15 and 16 almost do not differ from that of extracts number 3 and 6 , respectively.

The above-mentioned results were statistically confirmed and presented in Table 3.

Hypothesis testing was employed for establishing differences between two mean values of the total flavonoid content obtained over a short interval of time, in different days, for different ratio of bee bread to solvent, for different extracts, etc. In all cases the null hypothesis $\left(H_{0}\right)$ was tested that the difference is equal to zero, namely, two means were

Table 4. Antibacterial activity of the bee bread extracts with disc diffusion method in $\mathrm{mm}$.

\begin{tabular}{|c|c|c|c|c|c|c|}
\hline Sample & B. cereus & C. perfringens & S. aureus & H. influenzae & K. pneumoniae & S. enterica \\
\hline 1. & $14.3 \pm 2.1$ & $11.7 \pm 0.6$ & $12.0 \pm 2.0$ & $3.7 \pm 1.5$ & $2.0 \pm 1.0$ & $5.0 \pm 1.0$ \\
\hline 3. & $14.0 \pm 1.0$ & $11.7 \pm 1.5$ & $10.7 \pm 1.2$ & $4.0 \pm 1.0$ & $2.3 \pm 1.5$ & $2.0 \pm 1.0$ \\
\hline 4. & $14.3 \pm 2.1$ & $11.0 \pm 1.0$ & $13.0 \pm 1.0$ & $4.0 \pm 2.0$ & $2.0 \pm 1.0$ & $3.7 \pm 1.5$ \\
\hline 5. & $16.0 \pm 2.0$ & $12.7 \pm 1.1$ & $15.0 \pm 1.0$ & $5.0 \pm 1.0$ & $2.7 \pm 1.2$ & $5.0 \pm 1.0$ \\
\hline 6. & $15.7 \pm 2.1$ & $13.3 \pm 1.1$ & $15.0 \pm 1.0$ & $4.0 \pm 2.0$ & $2.0 \pm 1.0$ & $3.7 \pm 2.1$ \\
\hline 7. & $14.0 \pm 2.0$ & $14.3 \pm 2.1$ & $12.0 \pm 1.0$ & $4.0 \pm 1.0$ & $1.7 \pm 0.6$ & $4.3 \pm 0.6$ \\
\hline 8. & $12.3 \pm 0.6$ & $11.7 \pm 1.5$ & $9.0 \pm 1.0$ & $1.7 \pm 0.6$ & $2.3 \pm 0.6$ & $5.0 \pm 1.0$ \\
\hline 9. & $11.7 \pm 2.1$ & $11.7 \pm 0.6$ & $9.3 \pm 2.1$ & $3.0 \pm 1.0$ & $2.7 \pm 1.2$ & $4.7 \pm 2.5$ \\
\hline 10. & $13.3 \pm 1.5$ & $11.7 \pm 2.1$ & $13.7 \pm 1.5$ & $4.3 \pm 2.1$ & $2.7 \pm 0.6$ & $4.00 \pm 1.0$ \\
\hline 11. & $12.0 \pm 2.7$ & $14.3 \pm 2.1$ & $11.7 \pm 1.5$ & $4.0 \pm 1.0$ & $2.7 \pm 2.1$ & $3.7 \pm 2.1$ \\
\hline 12. & $13.3 \pm 1.5$ & $13.3 \pm 1.5$ & $11.0 \pm 1.0$ & $3.7 \pm 1.5$ & $3.0 \pm 1.0$ & $3.7 \pm 1.5$ \\
\hline 13. & $13.7 \pm 1.5$ & $13.0 \pm 1.0$ & $13.3 \pm 1.2$ & $3.3 \pm 0.6$ & $2.7 \pm 0.6$ & $5.0 \pm 1.0$ \\
\hline 14. & $12.3 \pm 2.1$ & $15.0 \pm 1.0$ & $11.7 \pm 1.5$ & $3.0 \pm 1.7$ & $2.7 \pm 1.2$ & $4.0 \pm 2.0$ \\
\hline 15. & $12.0 \pm 1.0$ & $15.0 \pm 3.0$ & $11.0 \pm 1.0$ & $3.0 \pm 1.0$ & $2.0 \pm 1.0$ & $3.3 \pm 1.5$ \\
\hline 16. & $15.0 \pm 1.0$ & $11.7 \pm 1.5$ & $12.7 \pm 1.5$ & $3.0 \pm 1.0$ & $2.3 \pm 1.5$ & $3.0 \pm 1.0$ \\
\hline DMSO & $\mathrm{ND}$ & $\mathrm{ND}$ & $\mathrm{ND}$ & $\mathrm{ND}$ & $\mathrm{ND}$ & $\mathrm{ND}$ \\
\hline GEN/AMP & $22.3 \pm 1.5$ & $22.7 \pm 1.2$ & $23.3 \pm 2.1$ & $23.3 \pm 1.5$ & $22.3 \pm 0.6$ & $21.7 \pm 2.1$ \\
\hline
\end{tabular}

ND - inhibition zones not detected; GEN - gentamicin; AMP - ampicillin 
assumed to be the same at $H_{0}\left(\mu_{1}=\mu_{2}\right)$. Sample data did provide evidence that the population means are not equal if we could reject the null hypothesis that the population means are equal, namely alternative hypothesis was opposite of the null hypothesis $\left(H_{a}\right.$ : $\left.\mu_{1} \neq \mu_{2}\right)$. For this purpose, it was necessary to pool sample variances by means of the following formula:

$$
S_{p}^{2}=\frac{\left(n_{1}-1\right) \cdot S D_{1}^{2}+\left(n_{2}-1\right) \cdot S D_{2}^{2}}{n_{1}+n_{2}-2}
$$

The statistical test was given by $t^{*}$ according to the following formula:

$$
t^{*}=\frac{\left(\bar{X}_{1}-\bar{X}_{2}\right)-\left(\mu_{1}-\mu_{2}\right)_{0}}{=\sqrt{\frac{S_{p}^{2}}{n_{1}}+\frac{S_{p}^{2}}{n_{2}}}}
$$

Which, when $H_{0}$ was true, is distributed as Student's $t$ with $n_{1}+n_{2}-2(3+3-2=4)$ degrees of freedom.

The conducted statistical analysis showed that the total flavonoid content in extracts with the ratios of $1: 5$ and $1: 10$ is statistically higher in comparison to the extracts with the ratios of $1: 10$ or $1: 20$, respectively, at the same maceration time (samples 16 and 15, 13 and 14, respectively). Not taking into account maceration time, the total flavonoid content in extracts with the ratios of $1: 5$ and $1: 10$ is sta- tistically higher in comparison to the extracts with the ratios of $1: 10$ or $1: 20$, respectively (samples 12 and 1, 1 and 5, respectively). The drawback of this study is different maceration times for some comparable extracts.

Beyond the aforementioned, from the statistical point of view, rutin is more convenient for analytical studies as it gives no statistically significant difference in the total flavonoid content for the same extract at the determination in different days (extracts 10-13), in the total flavonoid content of extracts prepared from the same bee bread sample with different maceration times (extracts 15 and 3 , 16 and 6). Moreover, the absorption maximum of the extracts is much closer to the ones of rutin that is more justified in the selection of a marker. These studies with statistical analysis provide interesting ideas for further research aiming at technological processes optimization, standardization of the method and validation of the analytical procedure of total flavonoid content determination and finding factors directed at a reduce of SD and, respectively, RSD.

In addition, compared to the Colombian Cundiboyacense Highland bee bread, Ukrainian samples contain less flavonoids that can be also explained by a lower content of Colombian bee bread in solvent $(1 \mathrm{~g}$ to $30 \mathrm{~mL})$ that facilitates better

Table 5. Anticandidal activity of the bee bread extracts with disc diffusion method in $\mathrm{mm}$.

\begin{tabular}{|c|c|c|c|}
\hline Sample & C. albicans & C. glabrata & C. tropicalis \\
\hline 1. & $6.7 \pm 1.5$ & $6.7 \pm 1.5$ & $5.3 \pm 0.6$ \\
\hline 3. & $6.7 \pm 1.5$ & $6.3 \pm 1.5$ & $4.0 \pm 1.0$ \\
\hline 4. & $7.7 \pm 0.6$ & $6.7 \pm 1.5$ & $6.0 \pm 1.0$ \\
\hline 5. & $5.0 \pm 1.0$ & $7.0 \pm 2.7$ & $5.0 \pm 1.0$ \\
\hline 6. & $5.7 \pm 1.5$ & $5.0 \pm 1.0$ & $7.0 \pm 1.0$ \\
\hline 7. & $6.7 \pm 1.5$ & $6.3 \pm 2.1$ & $5.0 \pm 1.0$ \\
\hline 8. & $6.0 \pm 1.0$ & $6.0 \pm 1.0$ & $6.7 \pm 1.5$ \\
\hline 9. & $5.0 \pm 1.0$ & $7.3 \pm 2.1$ & $5.7 \pm 1.5$ \\
\hline 10. & $5.3 \pm 1.5$ & $5.7 \pm 1.2$ & $5.7 \pm 2.1$ \\
\hline 11. & $5.7 \pm 1.2$ & $5.7 \pm 0.6$ & $5.3 \pm 0.6$ \\
\hline 12. & $6.3 \pm 1.2$ & $6.7 \pm 1.5$ & $5.0 \pm 1.0$ \\
\hline 13. & $5.3 \pm 0.6$ & $4.7 \pm 0.6$ & $4.7 \pm 0.6$ \\
\hline 14. & $8.0 \pm 1.0$ & $5.3 \pm 1.5$ & $5.3 \pm 0.6$ \\
\hline 15. & $5.0 \pm 1.0$ & $5.0 \pm 1.0$ & $6.7 \pm 1.5$ \\
\hline 16. & $5.0 \pm 1.0$ & $5.7 \pm 1.2$ & $6.7 \pm 1.5$ \\
\hline DMSO & $\mathrm{ND}$ & $\mathrm{ND}$ & $\mathrm{ND}$ \\
\hline AMP & $22.7 \pm 1.5$ & $23.67 \pm 1.5$ & $22.3 \pm 1.5$ \\
\hline
\end{tabular}

ND - inhibition zones not detected; AMP - ampicillin 
extraction of biologically active compounds but larger expenditures of ethanol (3). But Ukrainian bee bread samples contain more flavonoids compared with ones obtained in Western Slovakia (13). It is necessary to indicate that the results of different studies are compared hard as scientists employ different solvents, analytical procedures or even methods, ratios of bee bread to a solvent (methanol, ethanol), extraction techniques, analytical markers for calculation of the total flavonoid content and most studies are directed at determination of flavonoids in $1 \mathrm{~g}$ of bee bread $(2,3,5,6)$. This study is directed at the determination of the total flavonoid content in bee bread extracts prepared with different ratio of bee bread to $50 \%$ ethanol and different maceration times and study of precision parameters of the developed analytical procedure.

Finally, the results of this study also show that elaborated analytical procedure of the determination of the total flavonoid content is not precise enough as not in all the cases the results of determination in different days were in the range of $90-110 \%$ from an initial value that in line with the requirements of the Guideline on quality of herbal medicinal products/traditional herbal medicinal products. According to this Guide, in the case of a medicinal product containing a herbal preparation where con- stituents with known therapeutic activity are unknown, a variation in a marker content during the proposed shelf-life should be within $\pm 10 \%$ of the initial assay value (28). Further analytical and statistical studies are needed to enhance repeatability and intermediate precision of the developed analytical procedure.

The antibacterial activity of bee bread extract against Gram-positive and Gram-negative bacteria was shown in table 4 . The best antibacterial activity against Bacillus cereus $(16.00 \pm 2.00 \mathrm{~mm})$ was found in sample 5 (the sample of $20 \mathrm{~g} / 100 \mathrm{~mL}$ was macerated for 17 days). The strongest antibacterial activity of the bee bread extracts against Clostridium perfringens was found in samples 14 and 15 (15.0 \pm $1.0 \mathrm{~mm}$ and $15.0 \pm 3.0 \mathrm{~mm}$, respectively). Staphylococcus aureus was the most sensitive to samples 5 and $6(15.0 \pm 1.0 \mathrm{~mm})$. The best antibacterial activity of the extracts against Gram-positive bacteria was found for $B$. cereus.

The best antibacterial activity against Haemophilus influenzae $(5.0 \pm 1.0 \mathrm{~mm})$ was found in sample 5 (the sample of $20 \mathrm{~g} / 100 \mathrm{~mL}$ was macerated for 17 days). The strongest antibacterial activity against Klebsiella pneumoniae was found in sample $12(3.0 \pm 1.0 \mathrm{~mm})$. Salmonella enterica was the most sensitive to samples $1,5,8$, and $13(5.0 \pm 1.0$

Table 6. Antifungal activity of the bee bread extracts with disc diffusion method in $\mathrm{mm}$.

\begin{tabular}{|c|c|c|c|c|c|c|}
\hline Sample & A. clavatus & A. flavus & A. versicolor & P. expansum & P. chrysogenum & P. griseofulvum \\
\hline 1. & $2.0 \pm 0.0$ & $2.00 \pm 1.0$ & $1.7 \pm 0.6$ & $2.3 \pm 1.5$ & $2.3 \pm 1.5$ & $1.0 \pm 1.0$ \\
\hline 3. & $2.0 \pm 1.0$ & $2.33 \pm 1.5$ & $2.0 \pm 0.0$ & $1.7 \pm 0.6$ & $1.3 \pm 0.6$ & $1.7 \pm 0.6$ \\
\hline 4. & $2.7 \pm 1.2$ & $2.00 \pm 1.0$ & $2.0 \pm 1.0$ & $2.0 \pm 1.0$ & $1.3 \pm 0.6$ & $1.0 \pm 1.0$ \\
\hline 5. & $3.0 \pm 1.7$ & $2.33 \pm 0.6$ & $2.0 \pm 1.0$ & $2.3 \pm 0.6$ & $1.7 \pm 1.5$ & $1.3 \pm 0.6$ \\
\hline 6. & $1.3 \pm 1.2$ & $2.33 \pm 0.6$ & $2.0 \pm 1.0$ & $1.7 \pm 0.6$ & $2.7 \pm 1.5$ & $1.7 \pm 0.6$ \\
\hline 7. & $2.0 \pm 1.0$ & $2.00 \pm 0.0$ & $1.7 \pm 0.6$ & $3.0 \pm 1.0$ & $2.0 \pm 1.0$ & $1.3 \pm 1.5$ \\
\hline 8. & $2.7 \pm 2.5$ & $1.67 \pm 1.2$ & $1.0 \pm 1.0$ & $3.7 \pm 1.5$ & $2.7 \pm 1.2$ & $2.3 \pm 1.5$ \\
\hline 9. & $1.7 \pm 0.6$ & $2.67 \pm 1.2$ & $2.7 \pm 1.2$ & $2.0 \pm 1.0$ & $2.3 \pm 1.5$ & $2.3 \pm 0.6$ \\
\hline 10. & $2.3 \pm 0.6$ & $2.3 \pm 0.6$ & $1.7 \pm 1.5$ & $3.0 \pm 1.7$ & $2.3 \pm 0.6$ & $2.7 \pm 1.5$ \\
\hline 11. & $2.3 \pm 1.5$ & $1.7 \pm 0.6$ & $2.7 \pm 2.1$ & $3.7 \pm 2.1$ & $2.0 \pm 1.0$ & $2.3 \pm 0.6$ \\
\hline 12. & $2.0 \pm 1.0$ & $2.3 \pm 0.6$ & $1.7 \pm 0.6$ & $1.7 \pm 0.6$ & $2.0 \pm 0.0$ & $1.7 \pm 0.6$ \\
\hline 13. & $1.7 \pm 0.6$ & $1.3 \pm 0.6$ & $1.7 \pm 0.6$ & $2.0 \pm 1.0$ & $2.3 \pm 0.6$ & $1.7 \pm 0.6$ \\
\hline 14. & $2.3 \pm 0.6$ & $2.3 \pm 0.6$ & $2.0 \pm 1.0$ & $3.0 \pm 1.0$ & $2.3 \pm 1.5$ & $2.3 \pm 0.6$ \\
\hline 15. & $2.0 \pm 1.0$ & $1.7 \pm 0.6$ & $1.3 \pm 0.6$ & $2.3 \pm 0.6$ & $2.3 \pm 1.5$ & $1.3 \pm 0.6$ \\
\hline 16. & $1.7 \pm 0.6$ & $1.3 \pm 0.6$ & $2.0 \pm 1.0$ & $2.3 \pm 0.6$ & $2.0 \pm 1.0$ & $1.0 \pm 1.0$ \\
\hline DMSO & $\mathrm{ND}$ & $\mathrm{ND}$ & $\mathrm{ND}$ & $\mathrm{ND}$ & $\mathrm{ND}$ & $\mathrm{ND}$ \\
\hline GRI & $16.3 \pm 1.5$ & $17.3 \pm 1.2$ & $17.7 \pm 0.6$ & $16.3 \pm 0.6$ & $15.3 \pm 0.6$ & $15.3 \pm 1.2$ \\
\hline
\end{tabular}

ND - inhibition zones not detected; GRI - griseofulvin 
$\mathrm{mm})$. The best antibacterial activity of the extracts against Gram-negative bacteria was found for Salmonella enterica.

Our studies are in line with ones conducted by other scientists. In the study of Abouda et al. bee bread extracts in dilutions $1 / 2,1 / 4,1 / 8$ and 1/16 were tested by the agar well diffusion method on various strains of the bacteria including Escherichia coli, Staphylococcus aureus, Bacillus cereus, and Pseudomonas aeruginosa. Results revealed that most of the strains were inhibited by the dilution $1 / 2$ and $1 / 4$. The Gram-positive bacteria were more sensitive to bee bread compared to Gram-negative bacteria. All the samples showed strong antimicrobial activities on the bacterial strains, which were first tested for their resistance to antibiotics. The results showed that bee bread samples were more inhibitory than dried bee-pollen (10). The best antibacterial activity of bee bread with disc diffusion method was found against Bacillus thuringiensis CCM 19 and Staphylococcus aureus subsp. aureus CCM 4223 (2). Gram-positive bacteria (Bacillus cereus and Staphylococcus aureus) were more sensitive than Gramnegative bacteria (Escherichia coli and Salmonella) to all the extracts in the study carried by Md Akhir et al. (11).

The anticandidal activity of the bee bread extract was shown in table 5. The best anticandidal activity against Candida albicans $(8.0 \pm 1.0 \mathrm{~mm})$ was found in sample 14 (the sample of $10 \mathrm{~g} / 100 \mathrm{~mL}$ was macerated for 7 days). The strongest anticandidal activity of the bee bread extracts against Candida glabrata was found in sample $9(7.3 \pm 2.1$ $\mathrm{mm})$. Candida tropicalis was the most sensitive to sample $6(7.0 \pm 1.0 \mathrm{~mm})$. The best anticandidal activity was found against Candida albicans.

Antifungal activity of the bee bread extracts against Aspergillus and Penicillium genera was shown in table 6 . The best antifungal activity against Aspergillus clavatus $(3.0 \pm 1.7 \mathrm{~mm})$ was found in sample 5. The strongest antifungal activity of the bee bread extracts against Aspergillus flavus was found in sample $9(2.67 \pm 1.2 \mathrm{~mm})$. Aspergillus versicolor was the most sensitive to samples 9 and 11 ( $2.7 \pm 1.2$ and $2.7 \pm 2.1 \mathrm{~mm}$, respectively). The best antifungal activity against Penicillium expansum $(3.7 \pm 1.5$ and $3.7 \pm 2.1 \mathrm{~mm})$ was found in samples 8 and 11. Penicillium chrysogenum was the most sensitive to samples 6 and $8(2.7 \pm 1.5 \mathrm{~mm}$ and 2.7 $\pm 1.2 \mathrm{~mm}$, respectively). Penicillium griseofulvum was the most sensitive to sample $10(2.7 \pm 1.5 \mathrm{~mm})$. The best antifungal activity of the extracts against microscopic filamentous fungi was found for Penicillium expansum.

Correlations between the antibacterial activity for the most sensitive microorganisms and the content of bee bread in 50\% ethanol (Bacillus cereus, Clostridium perfringens, Staphylococcus aureus) were established and are presented in Figure 3.

The unexpectedly high and medium correlations ( $r=0.74$ and 0.61 , respectively) were obtained between the antimicrobial activity against Bacillus cereus and Staphylococcus aureus and the content of bee bread in $50 \%$ ethanol compared to a very weak correlation between the antimicrobial activity and the total flavonoid content $(\mathrm{r}=-0.177$ and 0.022 , respectively). The unexpected results were also obtained for Clostridium perfringens. In this case, a

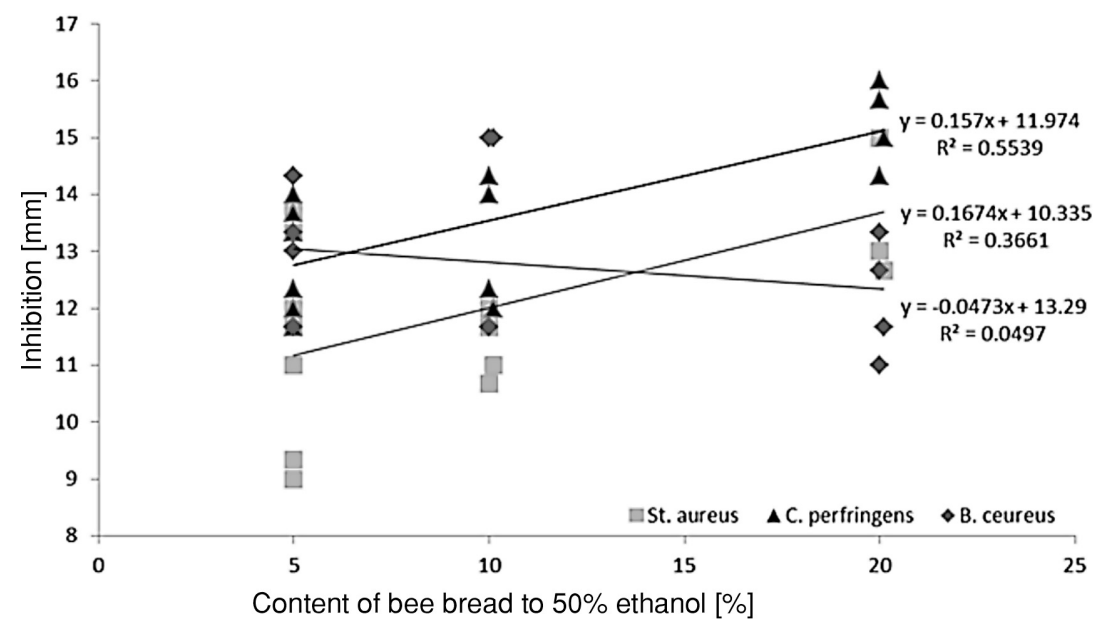

Figure 3. The dependence of the growth inhibition of Bacillus cereus, Clostridium perfringens and Staphylococcus aureus on the content of bee bread in $50 \%$ ethanol 
negative weak correlation between the antimicrobial activity and the content of bee bread in $50 \%$ ethanol was $r=-0.22$ compared to a weak correlation between the antimicrobial activity and the total flavonoid content $(\mathrm{r}=0.014)$.

The obtained results indicate that it is very important to employ extracts with a high content of bee bread in $50 \%$ ethanol during the development of bee bread extracts with the purpose of potential usage in medical practice.

\section{CONCLUSIONS}

The results of this investigation show that bee bread samples (obtained for technology with drying operation) from Ukraine are rich in flavonoids and compounds with antibacterial activity against Grampositive bacteria.

The total flavonoid content of the extracts mainly depended on a ratio of bee bread to $50 \%$ ethanol and technology of obtaining bee bread extracts. Moreover, the content of bee bread in 50\% ethanol correlated with the antimicrobial activity against Bacillus cereus and Staphylococcus aureus. The conducted statistical analysis showed that the total flavonoid content in the extracts with the ratios of $1: 5$ and $1: 10$ is statistically higher in comparison to the extracts with the ratios of $1: 10$ or $1: 20$, respectively. As the extracts with the ratio of bee bread to $50 \%$ ethanol of $1: 5$ and $1: 10$ are rich in flavonoids and possess good antibacterial activity against Gram-positive bacteria, the results of this study indicate the prospects of the usage of bread extracts as components of diet supplements and pharmaceutical products. Thus, the further studies will be directed at the development of extracts for diet supplements formulations and as well for the treatment of infectious diseases of the oral cavity, caused by Gram-positive bacteria, in the form of sprays and carrying out wider phytochemical, statistical technological and microbiological investigations with a larger number of extracts and pathogenic bacteria as well.

\section{Conflict of interests}

The authors have declared no conflict of interest.

\section{Acknowledgment}

The authors are thankful to Olena Tkachenko for providing the samples of bee bread.

Co-author Nataliia Hudz thanks the International Visegrad Fund for the scholarships for the research related to bee bread performed in the Slovak University of Agriculture in Nitra and University of Opole (Poland).

This work has been also supported by a grant of the European Community under project no. 26220220180: Building Research Centre "AgroBioTech" and by a grant of Slovak Research and development Agency No. VEGA 1/0411/17.

\section{REFERENCES}

1. Hudz N., Ivanova R., Brindza J., Grygorieva O., Schubertová Z. et al.: Potravinarstvo Slovak J. Food Sci. 11, 480 (2017).

2. Ivanišová E., Kačániová M., Frančáková H., Petrová J., Hutková J. et al.: Potravinarstvo Slovak J. Food Sci. 9, 592 (2015).

3. Zuluaga C.M., Serrato J.M., Quicazan M.C.: Chem. Eng. Trans. 43, 175 (2015).

4. Habryka C., Kruczek M., Drygaś B.: World Scientific News 48, 254 (2016).

5. Čeksterytė V., Kurtinaitienè B., Venskutonis P.R., Pukalskas A., Kazernavičiūtė R. et al.: Czech J. Food Sci. 34, 133 (2016).

6. Markiewicz-Żukowska R., Naliwajko S.K., Bartosiuk E., Moskwa J., Isidorov V. et al.: J. Apic. Sci. 57, 147 (2013).

7. Rzepecka-Stojko A., Stojko J., Kurek-Górecka A., Gorecki M., Kabala-Dzik A. et al.: Molecules 20, 21732 (2015).

8. Silva G.R., Natividade T.B., Camara C.A., Silva E.M.S., Santos F.A.R. et al.: Food Nutr. Sci. 5, 1015 (2014).

9. Barene I., Daberte I., Siksna S.: Theory and Practice in Medicine. 21, 16 (2015).

10. Abouda Z., Zerdani I., Kalalou I., Faid M., Ahami M.T.: Res. J. Microbiol. 6, 376 (2011).

11. Akhir R.A., Bakar M.F., Sanusi S.B.: AIP Conf. Proc. 1891, 020090 (2017).

12. Bogdanov S.: Bee Product Sci. Apr. 1 (2016).

13. Habryka C., Kruczek M., Drygaś B.: World Scientific News. 48, 254 (2016).

14. Baltrušaityte V., Venskutonisa P.R., Čeksterytė V.: Food Chem. 101, 502 (2007).

15. Fatrcová-Šramková K., Nôžková J., Márriássyová M., Kačániová M.: J. Environ. Sci. Health B 51, 176 (2016).

16. Baba S., Malik S.: J. Taibah Univ. Sci. 9, 449 (2015).

17. Chang C.C., Yang M.H., Wen H.W. Chern J.C.: J. Food Drug Anal. 10, 178 (2002).

18. Meda A., Lamien C.E., Romito M., Millogo J., Nacoulma O.G.: Food Chem. 91, 571 (2005). 
19. Zhang D.Y., Luo M., Wang W., Zhao Ch.J., Gu Ch.B. et al.: Food Chem. 141, 2231 (2013).

20. Ordońez A.A.L., Gomez J.D., Vattuone M.A., 1sla M.I.: Food Chem. 97, 452 (2006).

21. Pękal A., Pyrzyńska K.: Food Anal. Methods. 7 , 1776 (2014).

22. Ghasemi K., Ghasemi Y., Ehteshamnia A., Nabavi S.M., Nabavi S.F. et al.: J. Med. Plants Res. 5(7), 2228 (2011).

23. Ahmed S., Saeed-Ul-Hassan S., Islam M., Qureshi F., Waheed I. et al.: Acta Pol. Pharm. 74, 173 (2017).

24. Bühl A., Zöfel P.: SPSS Version 10. Einführung in die modern Datenanalyse unter Windows 7, Überarbeitete und erweiterte Auflage, Diasoft 2005.

25. Wayne W.D.: Biostatistics: A Foundation for Analysis in the Health Sciences $9^{\text {th }}$ Edition, USA 2009.
26. Sermukhamedova O.V., Sakipova Z.B., Ternynko I.I., Gemedzhieva N.G.: Acta Pol. Pharm. 74, 31 (2017).

27. Litvinenko V.I., Popova T.P., Popova N.V., Georgievskiy V.P.: Farmakom. 2, 22 (2016).

28. http://www.ema.europa.eu/docs/en_GB/document_library/Scientific_guideline/2011/09/WC 500113209.pdf (accessed on 01. 05. 2018).

29. Hudz N., Korzeniowska K., Wieczorek P.P., Schubertová Z., Brindza J. et al.: Agrobiodivers. Improv. Nutr. Health Life Qual. 1, 168 (2017).

30. Sobral F., Ricardo C., Calhelha R.C., Barros L., Dueńas M. et al.: Molecules 22, 248 (2017).

31. Tavdidishvili D., Khutsidze T., Pkhakadze M., Vanidze M., Kalandia A.: J. Chem. 8, 676 (2014).

32. Ursan A.C., Criste A.D., Dezmirean D.S., Margaoan R., Caeiro A., Campos M.G.: Molecules 23, 2491 (2018).

Received: 20.04.2018 